Commun. Fac. Sci. Univ. Ank. Serie B

V. 34. pp. 159-162 (1988)

\title{
THE REDUCTION OF SOME NITROBENZENES WITH ASCORBIC ACID
}

\author{
CELAL TÜZƯN, ATILLA ÖKTEMER, GÜLTEKIN YILDIZ, SEBLA BIRER \\ Department of Chemistry, Faculty of Science, University of Ankara
}

\section{ABSTRACT}

In this study the reduction of some nitrobenzenes with ascorbic acid were investigated. The substrates used in this study were 40 compounds containing electron donating groups such as methyl, methoxy, hydroxy amino and electron withdrawing groups such as halogens, formyl, carboxy and nitrile in addition one or two nitro groups. The nitro group was found to be totally reduced in substrates which gave reduction products. In the compounds containing dinitro groups it was observed that one of the nitro group was preferenteally reduced. The reduction products obtained were, 2- and 3- amino phenols, amino nitro halogenobenzenes, 2-, 3- and 4nitroanilines and amino nitro toluenes.

\section{INTRODUCTION}

There are various conditions and the reagents known for the reduction of aromatic nitro compounds. Some of these reagents cause preferential reduction (Samuelson, West, Wilson, Huber). The reduction of nitro groups gives monomolecular reduction products in acidie and bimolecular reduction products in alkaline media (Lapworth, Galbraith).

Ascorbic acid is well known to act as a reducing agent in biological systems. It has also been used to a limited extend as a reducing agent in some organic reactions (Wattok). Conrad and Eugen reduced picramic acid with ascorbic acid and obtained 2,6-diamino-4-nitrophenol in their patented Study (Eugen). In this study the reductions of mono and dinitrobenzenes with ascorbic acid were investigated.

ISSN 0570-1414 A. Üniv. Basımevi 


\section{RESULTS AND DICSUSSION}

The mono and dinitrobenzene derivatives studied in this study were as follows: nitrobenzene, 4-nitroanisole, 2-, 3-, 4- nitrophenols, 2 -, 3-, 4- nitro anilines; 2-, 3-, 4- nitro toluenos, 2-, 3-, 4- nitrobenzaldehydes, 3-, 4- nitro benzonitriles 3--, 4- nitro benzoic acids, 4- nitrochloro benzene, 4-nitrobromobenzene, 2-nitro iodobenzent, 1,2-,1,3-, 1,4dinitrobenzenes, 2,4-, 2,6- dinitroanilines, 2,4-, 2,5-, 2,6- dinitrob 3 henols, 2,3-, 3,4-, 2,6- dinitrotoluenes, 2,4-dinitro chloro, bromo and iodobenzenes and 2,4-, 3,4-3,5- dinitrobenzoic acids.

In the mononitro compounds which gave reduction products, the nitro group was reduced down to amine.

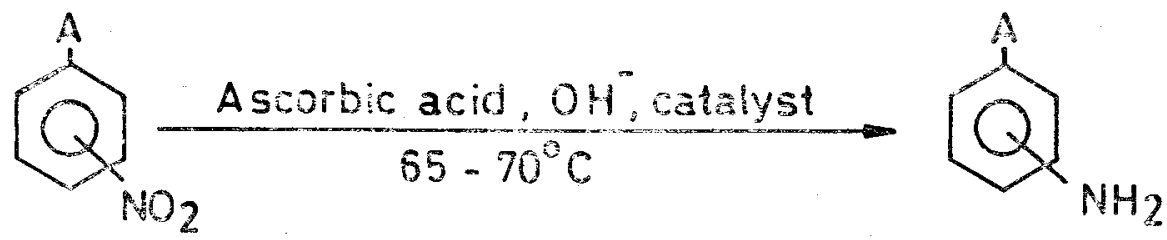

On the other hand in the dinitro compounds which gave reduction products, one of the nitro group was found to be reduced preferentaily down to amine.
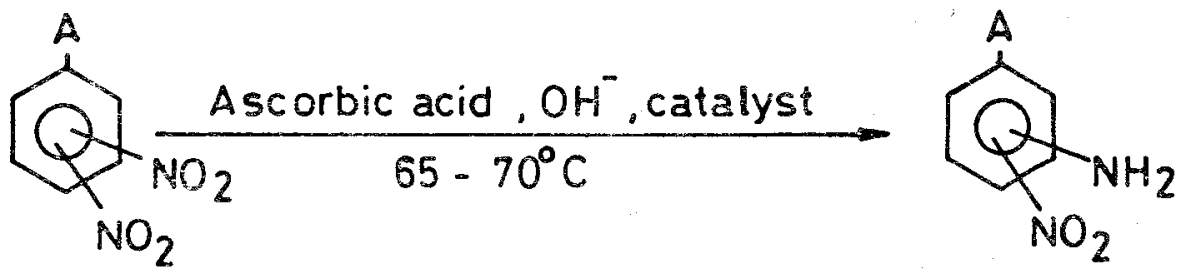

The reduction products and their yields were tabulated in Table $I$. According to it fifteen substrates gave reduction products. $\Lambda \mathrm{s}$ it is seen in the table three mononitro group gave reduction products while twelve of the total dinitro groups were found to be reducible.

Although it was not possible of isolate the reduction products, the qualitative tests and tho spectrophotometric analysis showed the partial reduction of nitrobenzene, 2-, 3-, 4- nitrotoluenes, 2-, 3-, 4- nitrobenzaldehydes, 3-, 4- nitrobenzoic acids and 2,6-dinitrophenol. The starting compounds were recovered in the experiments carried out with 
4-nitrophenol, 4-nitro anisole, mono and dinitrotoluenes and 3-, 4nitrobenzonitriles.

Table I

\begin{tabular}{|l|l|c|}
\hline \multicolumn{1}{|c|}{ Substrate } & \multicolumn{1}{|c|}{ Product } & Yild \% \\
\hline 2-Nitrophenol & 2-Aminophenol & 52 \\
3-Nitrophenol & 3-Aminophenol & 43 \\
2-Nitroiodobenzene & 2-Aminoiodobenzene & 17 \\
2,4-Dinitrophenol & 2-Amino-4-nitrophenol & 39 \\
2,5-Dinitrophenol & 2-Amino-5-nitrophenol & 40 \\
2,3-Dinitrotoluene & 2-Amino-3-nitrotoluene & 38 \\
3,4-Dinitrotoluene & 3,4-Diaminotoluene & 39 \\
2,6-Dinitrotoluene & 2-Amino-6-nitrotoluene & 39 \\
1,2-Dinitrobenzene & 1-Amino-2-nitrobenzene & 41 \\
1,3-Dinitrobenzene & 1-Amino-3-nitrobenzene & 37 \\
1,4-Dinitrobenzene & 1-Amino-4-nitrobenzene & 48 \\
1-Chloro-2,4-dinitrobenzene & 1-Chloro-2-amino-4-nitrobenzene & 20 \\
1-Bromo-2,4-dinitrobenzene & 1-Bromo-2-amino-4-nitrobenzene & 24 \\
1--Iodo-2,4-dinitrobenzene & 1-Iodo-2-amino-4-nitrobenzene & 45 \\
1-Bromo-3,5-dinitrobenzene & 1-Bromo-3-amino-5-nitrobenzene & 36 \\
\hline
\end{tabular}

Only one of the nitro groups was found to be reduced in the substrates carrying two nitro groups. Thus ascorbic acid acts as a preferential reducing agent. For instance in all halogeno dinitrobenzenes and dinitro phenols only the nitro group located in 2-position was observed to be reduced.

Only in 3,4-dinitrotoluene both nitro groups were ${ }^{r}$ reduced and gave 3,4 - diaminotoluene.

When nitroanilines and nitrophenols were compared it was found that none of the nitroanilines was reduced. This was the redults of the higher electron donating capability of the amino group.

Another interesting feature of the reductions of the aromatic nitor groups with ascorbic acid was, although the reactions were carried out in alkaline media, the reductions were monomolecular and nitro group was reduced down to amino group. On the other hand the oxidation potentials of $\mathrm{L}$-ascorbic acid and $\alpha$-D-glucose in DMSO were found to be -1.00 and 0.75 Volt respectively. Consequently ascorbic acid is a weaker reductant then glucose.

\section{EXPERIMENTAL}

Some of the substrates were synthesised with the proper methods in the literature. The others were used in their commercial forms without further purificiation. 
The IR and NMR spectra were recorded with Perkin-Elmer 477 spectrophotometer using $\mathrm{KBr}$ dics and Varian T60-A NMR spectrometer in $\mathrm{CDCl}_{3}$ respectively. The melting points were determined by Electrothermal capillary melting point apparatus without being corrected.

General Procedure: 0,03 moles of ascorbic acid, 0.01 moles of nitro compound and $10 \mathrm{ml}$ of water were put in a $250 \mathrm{ml}$ threenecked flask. The $\mathrm{pH}$ was kept in a range 7.5-8.0 by $1 \mathrm{M} \mathrm{NaOH}$ solution $(25-30 \mathrm{ml})$ throughout the experiment. $100 \mathrm{mg}$ of $\mathrm{FeSO}_{4}$ was employed as a catalyst. The mixture heated at $65-70^{\circ} \mathrm{C}$ for eight hours under nitrogen atmosphere. At the end the mixture was cooled down and the raw products were filtered of. It was recrystallised from ethanol.

\section{ACKNOWLEDGEMENT}

This study was supported by The Research Fund of Ankara University.

\section{REFERENCES}

SAMUELSON, G.S., et al., J. Am. Chem. Soc., 72, 3872 (1950)

WEST, R.W., J. Chem. Soc., 127, 494, (1925)

WILSON, J.W., et al., J. Org. Chem., 16, 792 (1951)

HUBER, D., ANDERMANN, G., LECLERE, G., Tetrahedron Lett., 29, 635 (1988)

LAPWORTH, A., and PEARSON, L.K., J. Chem. Soc,, 119, 765 (1920)

GALBRAITH, H.W., et al., J. Am. Chem. Soc., 73, 1323 (1951)

MATTOK, G.L., J. Chem. Soc., 4728 (1965)

EUGEN, K., THOMAS, C., (WELLA, A.G.), Chem. Abs., 6011 w (1985). 